\title{
The Effect of Dual N-Back Task Training on Phonological Memory Expansion in Adult EFL Learners at the Beginner Level
}

\author{
Mohammad Taghi Farvardin ${ }^{1}$, Akbar Afghari ${ }^{1} \&$ Mansour Koosha ${ }^{1}$ \\ ${ }^{1}$ Department of English Language, College of Foreign Languages, Khorasgan (Isfahan) Branch, Islamic Azad \\ University, Isfahan, Iran \\ Correspondence: Mohammad Taghi Farvardin, Department of English Language, College of Foreign Languages, \\ Khorasgan (Isfahan) Branch, Islamic Azad University, Isfahan, Iran. E-mail: mt.farvardin@khuisf.ac.ir
}

Received: July 7, 2014 Accepted: August 9, 2014 Online Published: August 14, 2014

doi:10.5539/elt.v7n9p137 URL: http://dx.doi.org/10.5539/elt.v7n9p137

\begin{abstract}
One of the most influential models of working memory (WM) is the one developed by Baddeley $(1986,2000$, 2003) which views WM comprising several components - a central executive, an episodic buffer, the visuo-spatial sketchpad, and the phonological loop. The phonological loop or phonological memory (PM) deals with the temporary storage of verbal and speech-based material. This study aimed to examine the effect of dual n-back task training on the expansion of PM capacity (PMC). One hundred twenty six adult EFL learners at the beginner level were randomly assigned to the control and experimental groups. The participants in the experimental groups were involved in dual n-back task training for six weeks. Nonword repetition and nonword recognition tests were used to measure PMC. The results showed that the training on the dual n-back task had a positive and significant effect on the expansion of the participants' PMC.
\end{abstract}

Keywords: working memory, phonological memory, dual n-back task, L2 learning

\section{Introduction}

One of the important factors in cognitive studies related to individual differences is working memory (WM). According to Baddeley (2003, p. 189), WM refers to the "temporary storage and manipulation of information that is assumed to be necessary for a wide range of complex cognitive activities". According to the Baddeley's WM model (2003), WM consists of the central executive, phonological loop, visuo-spatial sketchpad, and episodic buffer. The phonological loop has to do with the storage of verbal, speech-based material so that it can be immediately accessed and accurately repeated back aloud or silently rehearsed (Baddeley, 2000). Phonological loop has also been interchangeably referred to as phonological memory (PM) (Hummel \& French, 2010). Some scholars have highlighted PM as an important source of individual differences in L1 acquisition (e.g. Baddeley, 1986; Baddeley, 1996; Gathercole \& Baddeley, 1993) and in L2 learning (e.g., Harrington \& Sawyer, 1992; Papagno et al., 1991). According to Kormos and Sáfár (2008, p. 263), "as PM is responsible for remembering sequential information, its role in language learning is far greater than previously supposed."

Common tests used to measure PM are non-word repetition (hereafter NWRP) and non-word recognition (henceforth NWRC) tests. In NWRP, participants are asked to repeat non-words of various syllable lengths. As Hummel and French (2010, p. 374) put it, "the non-words generally consist of semantically empty items that follow the phonotactic structure of real words."

While working memory capacity (WMC) has long been reckoned to have a stringent limit (e.g., Cowan, 2001), growing evidence shows that WMC can be expanded though targeted training (e.g., Klingberg et al., 2005). The notion that training can significantly expand WMC has sparked great interest, and has given rise speculations that the cognitive benefits of WM training can be extensive (Jaeggi et al., 2008).

The evidence shows that WMC can be expanded and it suggests that similar effects may be possible for the PM subsystem of WM. A direct training effect on PM has in fact been reported in an L1 study with children conducted by Maridaki-Kassotaki (2002). They found that Greek-speaking children who received training on an NWRP test (15 minutes a day, four days a week) throughout their first school year outperformed matched children who did not receive such training when later tested on both NWRP and L1 reading tests. This study provides support for the notion that PMC can be expanded through specific training, with additional beneficial 
effects on reading skills.

As Morrison and Chein (2011) stated, generally the approaches to WM training are categorized based on their focus on domain-specific or domain-general components of the WM. One aspect of training studies examines strategy training which aim at increasing the use of domain-specific strategies that might allow trainees to remember increasing amounts of information of a particular type (e.g., McNamara \& Scott, 2001). On the other hand, core training studies involve repetition of tough WM tests designed to target domain-general WM mechanisms (e.g., Klingberg et al., 2002). One of the core training techniques is the dual n-back task. The $n$ refers to a number which can be adjusted to manipulate the difficulty of the task (Preece, 2011). Stimuli in the n-back task can be presented in either visual or auditory forms. In the dual n-back task, participants are required to simultaneously keep track of a visual stimulus and an auditory stimulus (Jaeggi et al., 2008). Jaeggi et al. (2008) trained participants for 25 minutes a day, for a period of 8, 12, 17 or 19 days through using a dual visualspatial $n$-back task. Based on participants' performance, the level of $n$ in the task adapted automatically. The participants in the Jaeggi et al. (2008) study were also administered traditional measures of WMC (reading span and digit span tasks) both before and after training. Compared to the untrained group, those who trained using the n-back improved significantly on the digit span but not on the reading span. Later, Jaeggi and her colleagues pursued their studies on the role of n-back training as a WM measure and found that n-back training is influential in expanding WMC (Jaeggi et al., 2010, 2011, 2014).

The present study aimed at examining the dual n-back task was administered to the experimental groups in order to see the task effects on the expansion of PMC. In effect, the researchers planned to examine the extent to which PMC can be expanded at the beginner level through using dual n-back task. Therefore, the following question was investigated in this study:

Does training on the dual n-back task lead to the expansion of phonological memory capacity in the adult EFL learners at the beginning level?

\section{Methods}

\subsection{Participants}

In the present study the participants' age ranged from 20 to 30 . The participants were selected from Iranian adult EFL learners at the beginner level. The present study took in 126 participants. The number of the male and female participants was 60 and 66, respectively. To assess their level of proficiency, all the participants were given the QPT v.1 (2001). Also, before data collection, the participants were informed that all the information collected during the study would be kept confidential. In the last week of the semester (Time 2), 9 participants were excluded from the study.

\subsection{Instruments}

\subsubsection{Nonword Repetition (NWRP) Test}

One of the most widely used tests of PMC is the NWRP, where participants have to repeat nonwords. The participants heard a list of 1-syllable nonwords and were asked to repeat them. Following the study carried out by Martin \& Ellis (2012, p. 385), "there were four lists at each of four lengths: three, four, five, and six words." The nonwords were taken from a stimulus pool of nonwords provided by Gathercole et al. (2001). All participants heard the lists, beginning with the shortest lists and continuing with lists of increasing length recorded by a male native English speaker. The maximum number of phonemes recalled on any one repetition set was calculated for each participant. The highest possible score for this test was 22 . Two raters scored the participants' responses to the NWRP to ensure the homogeneity of the calculated scores. The inter-rater reliability of the two sets of scores was $\mathrm{r}=.79(\mathrm{p}<.01)$ at Time 1 (week one) and $r=.74(p<.01)$ at Time 2 (week six).

\subsubsection{Nonword Recognition (NWRC) Test}

Two presentations of a list of nonwords were presented to the participants and afterwards, the participants decided whether they were the same or different (Afghari, Koosha, \& Farvardin, 2013). Four lists were used at each of four lengths: four, five, six and seven items. The maximum possible score for this test was 16 correct recognitions. The stimuli were recorded by an English male native speaker. Following Afghari et al. (2013,p.7) study, "two sequences of items were presented on each trial, with an inter-stimulus interval of $1.5 \mathrm{~s}$ separating the last item in the first presentation and the first item of the second presentation." 


\subsubsection{Quick Placement Test}

As Granpayeh (2003, p. 8) put it, "QPT is a test of English language proficiency developed by Oxford University Press and Cambridge ESOL to give teachers a reliable and time-saving method of finding a students' level of English." It is designed to calculate accurately English language learners' level of proficiency, from the beginner to the advanced level. QPT, which includes 60 items, takes 30 minutes to complete. According to Granpayeh (2003), the SEM of the test is around 4 and its reliability is close to 0.9 .

\subsubsection{Dual N-Back Task}

The experimental training task was dual $n$-back task executed using Brain Workshop (version 4.8.4), a freely available software (Jaeggi et al., 2008). The experimental groups performed the task four days per week for 6 weeks. In the experimental groups, the participants were instructed to complete the training sessions at the end of class for 15 minutes. The training sessions began with a main screen that displayed the number and proportion of correct and incorrect trials for both the verbal and spatial tasks for the previous block of trials, and the overall average $n$-level achieved during the training session (see Figure 1).

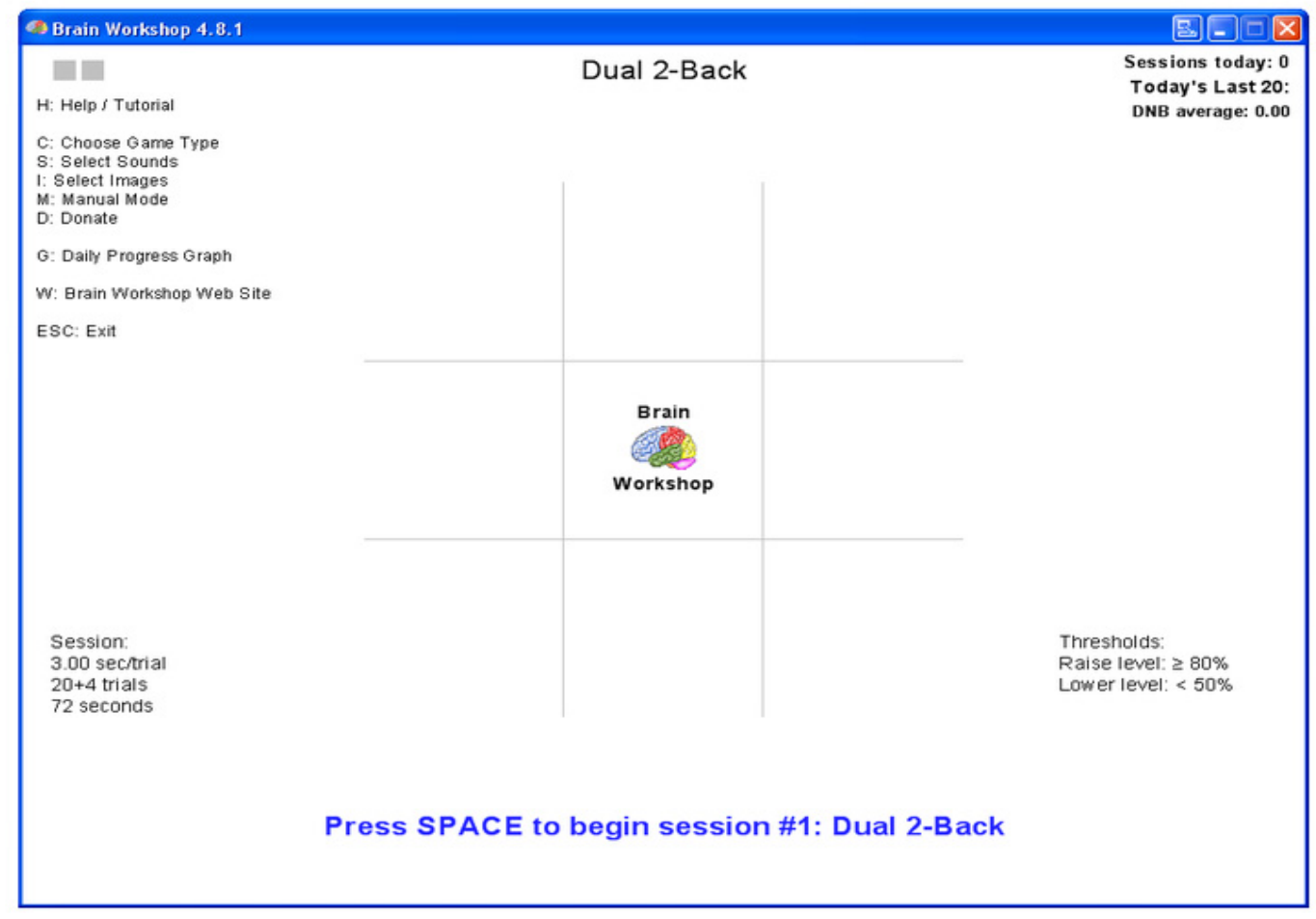

Figure 1. Screen capture of the brain workshop software (adapted from Hoskinson \& Toomim, 2008)

Scoring was calculated as the number of correct responses divided by the sum of the whole responses. If the average score was $80 \%$ or greater, than the $n$-level was increased by one. If the participants scored less than $50 \%$ on three blocks of trials, then the $n$-level was decreased by one for subsequent blocks.

\subsection{Data Collection Procedures}

First, all the participants were given the QPT v.1 (2001) in order to assess their level of proficiency. Based on the obtained scores on the QPT, the participants were assigned to the beginning proficiency level. The participants' classes were held four sessions a week, 24 sessions on aggregate. Each session lasted for two hours. The settings of the data collection were three English language institutes. The PM tests were measured during the first and last week of the English course. The allocated time for the NWRC and NWRP tests were 10 and 5 minutes, respectively.

In this study, two groups of participants were involved, control and experimental groups. The participants were randomly assigned to one of the two groups. The participants' classes for both the control and the experimental groups were held four sessions each week, 24 sessions on aggregate. Each session lasted for two hours. The participants in the experimental groups were involved in dual n-back task training which was expected to expand 
their PMC. The experimental groups were trained four days per week, 15 minutes per session.

In order to ensure understanding of the task, the researcher, first, explained the dual n-back task for the teachers and completed one round of "2-back" while the teachers observed. Following this, the teachers made the participants complete a round of '2-back' themselves, and encouraged the participants to continue completing rounds until they felt confident doing the activity. One session consisted of 15 rounds of the n-back, taking approximately 15 minutes. The total training period was 24 sessions over 6 weeks. The participants completed the training independently on the desktop computers available in their classes.

\subsection{Data Analysis}

In order to answer the research question, data from NWRP and NWRC tests were entered SPSS 21 (2012). In order to find out the effect of dual n-back task training on the expansion of PMC, independent samples and paired samples t-tests were calculated.

\section{Results}

The descriptive statistics are presented first, followed by the inferential statistics. The descriptive statistics for the PM tests in both control and experimental groups at Time 2 are presented in Table 1.

Table 1. Descriptive statistics of PM tests in control and experimental groups at time 2

\begin{tabular}{lllllll}
\hline & \multicolumn{3}{l}{ Control Groups } & \multicolumn{4}{c}{ Experimental Groups } \\
\hline Variables & $\mathrm{M}$ & $\mathrm{SD}$ & $\mathrm{N}$ & $\mathrm{M}$ & $\mathrm{SD}$ & $\mathrm{N}$ \\
NWRP Test & 15.50 & 1.72 & 60 & 16.98 & 1.89 & 57 \\
NWRC Test & 10.30 & 1.78 & 60 & 12.30 & 2.01 & 57 \\
\hline
\end{tabular}

Table 1 shows that the experimental groups had higher mean scores than control groups on both NWRP and NWRC tests. The mean scores of the experimental groups on NWRP and NWRC tests were higher than those of the control groups ( $\mathrm{Ms}=16.98$ and 12.30).

To answer the research question, a number of independent and paired samples t-tests were administered. T-tests were used to determine whether there were any significant differences between the control and experimental groups with regard to their performance on PM tests at Time 2, which is after the treatment. In other words, the effect of the treatment (dual $n$-back task) on the expansion of PMC was examined through t-tests. Table 2 shows the results of independent samples t-test for the beginners' PM scores between the control and experimental groups at Time 2 .

Table 2. Independent samples t-tests between control and experimental groups at time 2

\begin{tabular}{llllllll}
\hline Groups & & $\mathrm{N}$ & Mean & $\mathrm{SD}$ & $\mathrm{t}$ & $\mathrm{df}$ & $\mathrm{Sig}$ \\
\hline NWRP Test & Control & 60 & 15.50 & 1.72 & -4.43 & \multirow{2}{*}{115} & .00 \\
& Experimental & 57 & 16.98 & 1.89 & & & \\
NWRC Test & Control & 60 & 10.30 & 1.78 & -5.70 & \multirow{2}{*}{115} & .00 \\
& Experimental & 57 & 12.30 & 2.01 & & & \\
\hline
\end{tabular}

According to Table 2, there is a statistically significant difference between the control and experimental groups on both NWRP $(\mathrm{t}=-4.43, p<.05)$ and NWRC $(\mathrm{t}=-5.70, p<.05)$ tests. Moreover, the mean scores of the experimental groups on NWRP and NWRC tests were higher than those of the control groups. In other words, the experimental groups outperformed the control groups on the PM tests at Time 2 . To ensure the homogeneity of the participants at Time 1, independent samples t-tests for the beginners' PM scores between the control and experimental groups were computed (see Table 3 ). 
Table 3. Independent samples t-tests between control and experimental groups at time 1

\begin{tabular}{llllllll}
\hline Groups & & $\mathrm{N}$ & $\mathrm{Mean}$ & $\mathrm{SD}$ & $\mathrm{t}$ & $\mathrm{df}$ & $\mathrm{Sig}$ \\
\hline NWRP Test & Control & 66 & 15.50 & 1.91 & \multirow{2}{*}{1.39} & \multirow{2}{*}{124} & .17 \\
& Experimental & 60 & 14.70 & 1.71 & & & \\
NWRC Test & Control & 66 & 10.06 & 1.84 & .57 & \multirow{2}{*}{124} & .56 \\
& Experimental & 60 & 9.87 & 1.95 & & & \\
\hline
\end{tabular}

As shown in Table 3, the control and experimental groups are not significantly different on both NWRP $(\mathrm{t}=1.39$, $p>.05)$ and NWRC $(\mathrm{t}=.57, p>.05)$ tests. In other words, the control and experimental groups did not perform differently on the PM tests at Time 1. Paired-samples t-tests were used to see whether there was a statistically significant difference in the mean scores for Time 1 (prior to the treatment) and Time 2 (after the treatment) of the same group (see Table 4).

Table 4. Paired samples t-tests for comparing the pre-test and post-test results of PM tests in control group

\begin{tabular}{llllllll}
\hline Groups & & $\mathrm{N}$ & Mean & $\mathrm{SD}$ & $\mathrm{t}$ & $\mathrm{df}$ & $\mathrm{Sig}$ \\
\hline Pair 1 & NWRP Pre-test & 60 & 15.15 & 1.99 & \multirow{2}{*}{-3.08} & 59 & .12 \\
& NWRP Post-test & 60 & 15.50 & 1.72 & & & \\
Pair 2 & NWRC Pre-test & 60 & 9.93 & 1.81 & \multirow{2}{*}{-2.30} & 59 & .25 \\
& NWRC Post-test & 60 & 10.30 & 1.77 & & & \\
\hline
\end{tabular}

Table 4 illustrates that there is no significant difference between the mean scores of the control groups before and after the treatment (NWRP test: $\mathrm{t}=-3.08, p>.05$; NWRC test: $\mathrm{t}=-2.30, p>.05$ ). Table 5 displays the results of the comparison between the mean scores of the experimental group in pre-test and post-test.

Table 5. Paired samples t-tests for comparing the pre-test and the post-test results of PM tests in experimental group

\begin{tabular}{llllllll}
\hline Groups & & $\mathrm{N}$ & $\mathrm{Mean}$ & $\mathrm{SD}$ & $\mathrm{t}$ & $\mathrm{df}$ & $\mathrm{Sig}$ \\
\hline Pair 1 & NWRP Pre-test & 57 & 14.65 & 1.74 & \multirow{2}{*}{-13.69} & \multirow{2}{*}{56} & \multirow{2}{*}{.00} \\
& NWRP Post-test & 57 & 16.98 & 1.89 & & & \\
Pair 2 & NWRC Pre-test & 57 & 9.89 & 1.96 & -12.96 & 56 & .00 \\
& NWRC Post-test & 57 & 12.30 & 2.01 & & & \\
\hline
\end{tabular}

Table 5 depicts that there is a significant difference between the mean scores of the experimental groups in pre-test and post-test (NWRP test: $\mathrm{t}=-13.69, p<.05$; NWRC: $\mathrm{t}=-12.96, p<.05$ ). In other words, the treatment (dual $n$-back task) had a significant effect on the expansion of the beginners' PMC.

\section{Discussion and Conclusion}

The current research examined the issue that whether training on the dual $n$-back task leads to the expansion of PMC (measured by NWRP and NWRC recognition tests) in adult EFL learners at the beginner level. The results showed that the training on the dual $n$-back task had a positive and significant effect on the expansion of the participants' PMC. The results are in line with Jaeggi et al. (2008) and Klingberg et al. (2002) in that they argued that dual $n$-back task training can enhance WMC and other related subcomponents of WM. In this study, PM is considered as a subcomponent of WM. Moreover, Buschkuehl and Jaeggi (2010) argued that a training task should be adaptive in difficulty so that the difficulty level of the training task matches the skill level of each participant, thus providing a relatively similar high level of training demand to all participants. The results also showed that dual n-back task can be a practical and useful task for expanding the WM and consequently PMC of the adult EFL beginners. 
The results of the present study showed that the training on the dual n-back task had a positive and significant effect on the expansion of the participants' PMC. It can be concluded that PM has an important role in adults' L2 learning, and the training on the dual n-back task can significantly affect the expansion of the participants' PMC. Teachers can help students expand their PMC by applying various strategies and techniques and see the effects of this expansion on the students' L2 learning. Furthermore, as it seems that there are strong associations between PM and L2 knowledge, the first step should be to provide aid for those with poor PMC. The current understanding is that little can be done to expand poor PM but that there are ways to remove this obstacle in L2 learning as much as possible. This goal can be achieved by the teacher favoring strategies and techniques that do not require learners to rely heavily on their PM.

\section{References}

Afghari, A., Koosha, M., \& Farvardin, M. T. (2013). The relationship between L2 grammatical knowledge and phonological memory in adult male EFL learners at different language proficiency levels. International Journal of Language Learning and Applied Linguistics World, 4(4), 1-12.

Baddeley, A. D. (1986). Working memory. Oxford: Oxford University Press.

Baddeley, A. D. (1996). The concept of working memory. In S. E. Gathercole (Ed.), Models of short-term memory (pp. 1-27). East Sussex: Psychology Press.

Baddeley, A. D. (2000). The episodic buffer: A new component of working memory? Trends in Cognitive Sciences, 4, 417-422. http://dx.doi.org/10.1016/s1364-6613(00)01538-2

Baddeley, A. D. (2003). Working memory and language: An overview. Journal of Communication Disorders, 36(3), 189-208. http://dx.doi.org/10.1016/s0021-9924(03)00019-4

Buschkuehl, M., \& Jaeggi, S. M. (2010). Improving intelligence: A literature review. Swiss Medical Weekly, 140, 266-272. http://dx.doi.org/smw-12852

Cowan, N. (2001). The magical number 4 in short-term memory: A reconsideration of mental storage capacity. Behavioral and Brain Sciences, 24, 87-185. http://dx.doi.org/10.1017/s0140525x01003922

Gathercole, S. E., \& Baddeley, A. D. (1993). Working memory and language. Hillsdale, NJ: Lawrence Erlbaum.

Gathercole, S. E., Pickering, S. J., Hall, M., \& Peaker, S. M. (2001). Dissociable lexical and phonological influences on serial recognition and serial recall. The Quarterly Journal of Experimental Psychology, 54A(1), 1-30. http://dx.doi.org/10.1080/02724980042000002

Granpayeh, A. (2003). A quick review of the English Quick Placement Test. Research Notes, 12, 8-10.

Harrington, M., \& Sawyer, M. (1992). L2 working memory capacity and L2 reading skill. Studies in Second Language Acquisition, 14, 25-38. http://dx.doi.org/10.1017/s0272263100010457

Hoskinson, P., \& Toomim, J. (2008). Brain Workshop (Version 4.8.1) [Computer Software].

Hummel, K. M., \& French, L. M. (2010). Phonological memory and implications for the second language classroom. The Canadian Modern Language Review, 66(3), 371-391. http://dx.doi.org/10.3138/cmlr.66.3.371

IBM Corp. Released. (2012). IBM SPSS Statistics for Windows (Version 21.0). Armonk, NY: IBM Corp.

Jaeggi, S. M., Buschkuehl, M., Jonides, J., \& Perrig, W. J. (2008). Improving fluid intelligence with training on working memory. Proceedings of the National Academy of Sciences, 105, 6829-6833. http://dx.doi.org/10.1073/pnas.0801268105

Jaeggi, S. M., Buschkuehl, M., Jonides, J., \& Shah, P. (2011). Short-and long-term benefits of cognitive training. Proceedings of the National Academy of Sciences, 108(25). http://dx.doi.org/10.1073/pnas.1103228108

Jaeggi, S. M., Buschkuehl, M., Perrig, W. J., \& Meier, B. (2010). The concurrent validity of the N-back task as a working memory measure. Memory, 18(4), 394-412. http://dx.doi.org/10.1080/09658211003702171

Jaeggi, S. M., Buschkuehl, M., Shah, P., \& Jonides, J. (2014). The role of individual differences in cognitive training and transfer. Memory and Cognition, 42(3), 464-480. http://dx.doi.org/10.3410/f.718143662.793485334

Klingberg, T., Fernell, E., Olesen, P. J., Johnson, M., Gustafsson, P., Dahlstrom, K., \& Westerberg, H. (2005). Computerized training of working memory in children with ADHD-A randomized, controlled trial. Journal of the American Academy of Child and Adolescent Psychiatry, 44, 177-186. http://dx.doi.org/10.1097/00004583-200502000-00010 
Klingberg, T., Forssberg, H., \& Westerberg, H. (2002). Training of working memory in children with ADHD. Journal of Clinical and Experimental Neuropsychology, 24, 781-791. http://dx.doi.org/10.1076/jcen.24.6.781.8395

Kormos, J., \& Sáfár, A. (2008). Phonological short-term memory, working memory and foreign language performance in intensive language learning. Bilingualism: Language and Cognition, 11(2), 261-271. http://dx.doi.org/10.1017/s1366728908003416

Maridaki-Kassotaki, K. (2002). The relation between phonological memory skills and reading ability in Greek-speaking children: Practical applications. European Journal of the Psychology of Education, 1, 63-73. http://dx.doi.org/10.1007/bf03173205

Martin, K. I., \& Ellis, N. (2012). The roles of phonological short-term memory and working memory in L2 grammar and vocabulary learning. Studies in Second Language Acquisition, 34, 379-413. http://dx.doi.org/10.1017/s0272263112000125

McNamara, D. S., \& Scott, J. L. (2001).Working memory capacity and strategy use. Memory and Cognition, 29, 10-17. http://dx.doi.org/10.3758/bf03195736

Morrison, A. B., \& Chein, J. M. (2011). Does working memory training work? The promise and challenges of enhancing cognition by training working memory. Psychonomic Bulletin \& Review, 18(1), 46-60. http://dx.doi.org/10.3758/s13423-010-0034-0

Papagno, C., Valentine, T., \& Baddeley, A. D. (1991). Phonological short-term memory and foreign-language vocabulary learning. Journal of Memory and Language, 30, 331-347. http://dx.doi.org/10.1016/0749-596x(91)90040-q

Preece, D. (2011). The effect of working memory (n-back) training on fluid intelligence. Honours thesis, Edith Cowan University, Perth Australia.

Quick Placement Test. (2001). Oxford: Oxford University Press.

\section{Copyrights}

Copyright for this article is retained by the author(s), with first publication rights granted to the journal.

This is an open-access article distributed under the terms and conditions of the Creative Commons Attribution license (http://creativecommons.org/licenses/by/3.0/). 